\title{
The role of familiarity in recognition
}

\author{
ROBERT L. GREENE \\ Case Western Reserve University, Cleveland, Ohio
}

\begin{abstract}
The role of familiarity in recognition was investigated by having subjects study a list of stimuli, some of which had been presented earlier in the experiment. The number of positive responses, both to targets and distractors, increased as a result of this familiarization process. This familiarization process had a greater effect on false alarms than on hits, so that recognition accuracy was lower for familiar stimuli than for relatively novel stimuli. This pattern of results differs from that found in most experiments studying the effects of linguistic word frequency on recognition.
\end{abstract}

How is recognition affected by the familiarity of the stimuli? This issue has been studied recently by two different sets of investigators, who found different patterns of results (Maddox \& Estes, 1997; Tulving \& Kroll, 1995). Both sets of investigators used what Maddox and Estes called a three-phase design. In the first phase, subjects are exposed to stimuli. In the second phase, subjects receive a study list that contains these preexposed stimuli (as well as possibly other stimuli that have not been presented earlier in the experiment). In the third phase, subjects receive a recognition test on the study list.

Tulving and Kroll (1995) had four subjects make selfpaced living/nonliving judgments to a set of words. After a short break, subjects made living/nonliving judgments to a second set of words. This second set contained some words that had been in the first set, as well as some new words. Finally, subjects received a recognition test and were asked to respond positively only to words that had been on the second list. Tulving and Kroll found that recognition accuracy was lower for familiar stimuli (i.e., those that had been included on the first set) than for novel stimuli (i.e., those that had not been included on the first set). In particular, hits were lower, and false alarms higher, for the familiar stimuli than for the novel stimuli, thus exhibiting a pattern referred to as the mirror effect (Glanzer \& Adams, 1985). Tulving and Kroll noted that their pattern resembled that found in studies comparing recognition for words varying in frequency of occurrence in the language and suggested that both reflect the operation of a novelty-assessment mechanism, which prevents familiar stimuli from being extensively processed.

Tulving and Kroll's (1995) data, and particularly their finding that an experimental manipulation of familiarity decreased hits and increased false alarms, are notable for several reasons. First, if experimental familiarization really has effects similar to those of linguistic frequency, it

Neal Kroll, Todd Maddox, and John Wixted provided helpful comments on an earlier version. Address correspondence to R. L. Greene, Department of Psychology, Case Western Reserve University, Cleveland, OH 44106 (e-mail: rlg2@po.cwru.edu). would be strong evidence that the latter truly is due to the frequency of the stimuli per se and not to other word characteristics that are correlated with word frequency. Second, the finding that the hit rate is lower for familiar words than for novel words appears difficult to reconcile with the assumption underlying many formal models of recognition (e.g., Estes, 1994; Gillund \& Shiffrin, 1984; Hintzman, 1988) that recognition decisions are largely driven by familiarity (usually defined as the overall similarity between a test stimulus and the contents of memory); these models assume that the probability of a positive response increases as a monotonic function of familiarity.

However, Maddox and Estes (1997) reported a pair of experiments that found very different results from those of Tulving and Kroll (1995). Maddox and Estes manipulated the frequency of nonword stimuli from one to four presented at a 1.5 -sec pace in the first phase of a threephase design. Some of these stimuli were presented again in the second phase. On the recognition test administered in the third phase, both hits and false alarms increased as Phase 1 frequency increased from one to four, resulting in little change in accuracy; Maddox and Estes referred to this pattern (with a manipulation affecting hit rates and false alarm rates in the same direction) as a concordant pattern. Not surprisingly, Maddox and Estes's conclusions were largely opposite to those that could be drawn from the Tulving and Kroll study. Maddox and Estes concluded that the effects of experimental familiarization differed from those of linguistic frequency, so that the latter probably did not reflect frequency per se and the basic pattern of results was consistent with the assumptions of global-matching models of recognition.

Maddox and Estes (1997) noted that the design of the Tulving and Kroll (1995) study was the one closest to theirs in the literature. They suggested that the discrepancy in results reflected a lack of control of the learning conditions in the Tulving and Kroll study, highlighting two factors in particular - namely, that presentation of stimuli was subject paced and that words were used as stimuli. These two factors could have led subjects to follow a strategy of devoting more rehearsal to the less familiar items during presentation of the second list. 
The experiments reported here addressed the question of the effects of familiarity on recognition memory. In Experiment 1, all subjects received two lists and were then tested on the second list. Two manipulations were varied across subjects. The first was the nature of the tobe-remembered stimuli (words, as had been used by Tulving \& Kroll [1995], or digit triads, which had been used by Maddox \& Estes [1997]). The second was control of the presentation of the study (i.e., second) list (subject paced or a constant 1.5 -sec rate), which would influence the opportunity for subjects to rehearse items differentially on the second list.

\section{EXPERIMENT 1}

\begin{abstract}
Method
Subjects. Sixty-four students in introductory psychology classes participated to fulfill a course requirement.

Design. The structure of the experiment was (1) a familiarization series, in which stimuli could occur four times, twice, or once; (2) a study list which contained half of the items from the familiarization series and an equal number of unfamiliarized stimuli, all occurring once; and (3) a recognition test, in which subjects were asked to give positive responses only to items from the study list. There were two repeated measures factors: familiarization frequency (number of occurrences on the familiarization series: four, two, one, or none) and study (whether or not an item had appeared on the study list). There were two between-subjects manipulations: stimulus type (common nouns [with rates of occurrence of at least 50 times per million words, according to Thorndike \& Lorge, 1944] and digit triads constructed randomly) and presentation mode of the study list (1.5-sec rate or self-paced mode, with the subject pressing a key to advance to the next item).
\end{abstract}

Procedure. Subjects were asked to study a series of items (the familiarization series) for an unspecified memory test. This familiarization series consisted of 30 items presented four times, 30 items presented twice, and 30 items presented once, as well as 5 once-occurring buffers at the beginning and 5 at the end of the list, for a total of 220 presentations. This series was presented on a computer screen at a $1.5-\mathrm{sec}$ rate. Immediately after completion of the familiarization series, subjects were asked to study another set of items (the study list) for an unspecified memory test. The study list was presented on a computer screen, at a $1.5-\mathrm{sec}$ rate for half of the subjects and in a self-paced mode for the others. The study list consisted of 60 critical words (equal numbers of which had occurred four times, twice, once, or not at all in the familiarization series) as well as 10 untested buffers. All items occurred once on the study list.

After the study list was completed, subjects were given a memory test in which they were asked to give a positive response only to items that had occurred on the study (i.e., second) list. This test contained 120 items, with 15 items falling in each of eight conditions. The eight conditions resulted from the crossing of four familiarization frequencies (four, two, one, or zero) with two study conditions (studied or nonstudied on the study list). In all of the experiments reported here, assignment of stimuli to familiarization frequency and study condition was randomized separately for each subject.

\section{Results}

Table 1 displays the proportion of positive responses given to items that were studied or nonstudied on the study (i.e., second) list as a function of familiarization frequency and stimulus type (word or digit triad). Clearly, familiarity increased the number of positive responses, a
Table 1

Proportion of Test Stimuli Judged to be From the Study List Familiarization Frequency

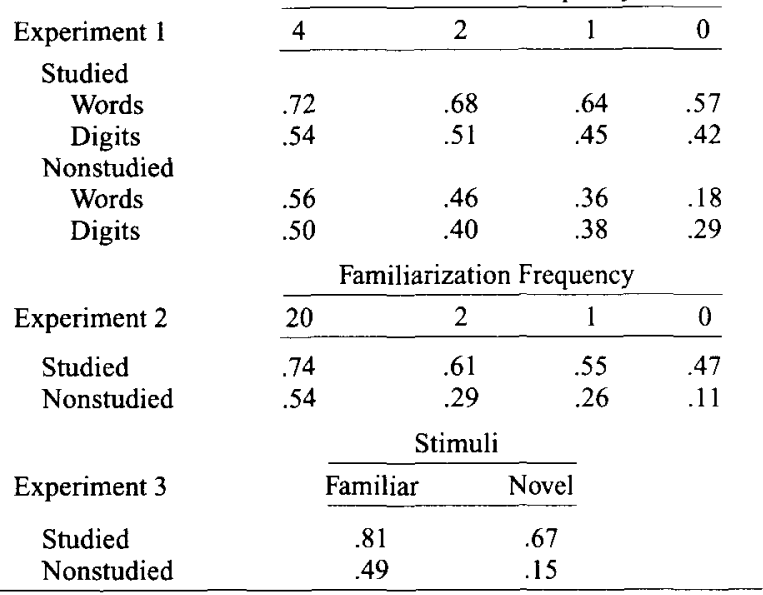

conclusion in general agreement with Maddox and Estes (1997). This effect was present for both studied and nonstudied items, although the effect appears somewhat larger in the latter case. Whether presentation on the study list was self-paced or computer paced did not interact with any of the other variables, so the proportions shown in Table 1 were collapsed across this variable. The type of stimulus (digit triads or words) did have an effect, but the overall pattern of familiarity increasing the number of positive responses was present for both stimulus classes.

An analysis of variance was conducted on the number of positive responses as a function of the within-subjects variables of familiarization frequency (occurrences on the familiarization series: four, two, one, or none) and study (whether or not an item occurred on the study list), as well as the between-subjects variables of stimulus type (word or digit triad) and pacing (self-paced or 1.5sec rate). The critical findings were the significant effect of familiarization frequency $\left[F(3,180)=69.86, M S_{\mathrm{e}}=\right.$ 3.57] and the significant interaction of familiarization frequency with study $\left[F(3,180)=14.44, M S_{\mathrm{e}}=2.38\right]$, showing that nonstudied items were more affected by familiarization frequency than were studied items. There was also a significant three-way interaction among familiarization frequency, study, and stimulus type $[F(3,180)=$ $\left.3.75, M S_{\mathrm{e}}=2.38\right]$, suggesting that accuracy in memory for words was affected more by familiarization frequency than was accuracy for digit triads. For completeness, it should be noted that the overall number of positive responses was significantly affected by study, stimulus type, and pacing, and there was a significant interaction between study and stimulus type (suggesting greater accuracy for words than for digit triads).

Because the effect of familiarization frequency was greater on nonstudied items than on studied items, accuracy declined as a function of familiarity. A similar conclusion is reached if one calculates $d^{\prime}$ scores. As familiar- 
ization frequency decreases from four to two to one to zero, mean $d^{\prime}$ scores increase from 0.30 to 0.44 to 0.47 to 0.83 ; this effect was significant $\left[F(3,186)=14.67, M S_{\mathrm{e}}=0.22\right]$.

\section{EXPERIMENT 2}

In Experiment 1, subjects were more likely to give a positive response to a familiar test stimulus than to a novel one. This stands in contrast to the results of Tulving and Kroll (1995), who found that the probability of a positive response decreased for studied items as a function of familiarity. However, they had used a higher number of familiarization exposures (a total of six) than had been used in Experiment 1. Possibly, the function relating hits to familiarity was nonmonotonic, so that a very high level of familiarity must be employed before the hit rate decreases. This was investigated by having items on the familiarization series occur 20 times, 2 times, or 1 time. Because type of stimulus and item pacing had not appeared critical in Experiment 1, in Experiment 2 we used only word stimuli, and all items appeared at a 1.5sec rate.

\section{Method}

Subjects. Twenty-four students from introductory psychology classes participated to fulfill a course requirement.

Procedure. The procedure was identical to that used in the condition of Experiment 1 employing word stimuli and a computercontrolled $1.5-\mathrm{sec}$ presentation rate, except that the familiarization series now contained 30 words occurring 20 times each, 30 words occurring 2 times each, and 30 words occurring once, along with 10 buffer words, for a total of 700 presentations. As in Experiment 1 , each item occurred only once on the study list, and the subsequent test required subjects to give positive responses only to items included on the study list.

\section{Results}

As shown in Table 1, familiarity increased both hits and false alarms, with the latter being somewhat more affected than the former. An analysis of variance was conducted on the number of positive responses. There were significant main effects of familiarization frequency $\left[F(3,39)=83.32, M S_{\mathrm{e}}=2.80\right]$ and study $[F(1,23)=$ $\left.158.88, M S_{\mathrm{e}}=5.78\right]$. There was also a significant interaction between familiarization frequency and study $\left[F(3,69)=3.78, M S_{\mathrm{e}}=3.26\right]$, indicating that nonstudied items were more affected by familiarization frequency than were studied items. This pattern is the same as that found in Experiment 1. The fact that false alarms were more affected by familiarization frequency than were hits suggests that recognition accuracy was impaired by familiarity. For familiarization frequencies of $20,2,1$, and 0 , mean $d^{\prime}$ scores were $0.58,0.89,0.74$, and 1.17; the effect of familiarization frequency on $d^{\prime}$ was significant $\left[F(3,51)=3.70, M S_{\mathrm{e}}=0.30\right]$.

\section{EXPERIMENT 3}

In Experiments 1 and 2, subjects did not have to perform any sort of orienting task during the presentation of the familiarization series or the study list. However, Tulving and Kroll (1995) had their subjects perform living/ nonliving judgments during presentation of both the familiarization series and the study list. In Experiment 3, we included this in the procedure so that it could be determined whether this was critical.

\section{Method}

Subjects. Twenty-four students from introductory psychology classes participated to fulfill a course requirement.

Procedure. In the first phase, subjects saw a set of 80 words, each presented four times, and had to make a self-paced living/ nonliving judgment about each one on each presentation. Thus, there were 320 presentations in this sequence. Then, there was a 5 -min interpolated typing task. After this, subjects saw a set of 80 words, all presented once, and had to make self-paced living/nonliving judgments for each of them; of these 80 words, half had been shown in the first phase, and half had not. Then, subjects were given a recognition test in which they were shown 160 words. This test consisted of 40 words shown in both phases, 40 shown in the first phase and not in the second, 40 shown in the second phase and not in the first, and 40 not previously shown in the experiment. The subjects were asked to give positive responses to those shown in the second phase.

\section{Results}

In Experiment 3, we found a pattern of results similar to those found in the first two experiments. Once again, the proportion of positive responses was higher for familiar stimuli than for novel stimuli, with this difference being greater for nonstudied items than for studied items. Table 1 displays the proportion of positive responses as a function of familiarity (inclusion on the familiarization series) and study (studied or nonstudied on the study list). An analysis of variance conducted on the number of positive responses revealed significant effects of familiarization $\left[F(1,23)=266.79, M S_{\mathrm{e}}=25.55\right]$ and study $\left[F(1,23)=405.41, M S_{\mathrm{e}}=5.65\right]$, as well as a significant interaction between these factors $\left[F(1,23)=30.01, M S_{\mathrm{e}}=\right.$ 12.39]. Because familiarity had a greater effect on false alarms than on hits, there was a significant difference between the mean $d^{\prime}$ scores for familiar (0.52) and novel $(1.12)$ items $\left[F(1,23)=29.11, M S_{\mathrm{e}}=0.15\right]$.

\section{GENERAL DISCUSSION}

In all three experiments, subjects were more likely to give positive responses to familiar stimuli than to novel stimuli. This effect followed what Maddox and Estes (1997) termed a concordant pattern, with both hits and false alarms increasing as a function of familiarity. This result is in agreement with the results of Maddox and Estes and is also consistent with the general assumption in global matching models that recognition decisions are largely driven by familiarity.

In all three experiments, experimental familiarity increased both hits and false alarms. This contrasts with studies of the effects of linguistic word frequency on recognition, where hits decrease and false alarms increase as a function of word frequency. Thus, the results here are in agreement with Maddox and Estes's (1997) argument that linguistic word frequency effects in recognition 
are not due to frequency of occurrence per se but may reflect other properties that are correlated with linguistic frequency.

Tulving and Kroll (1995) tested only 4 subjects in their study. It is possible that the particular pattern that they found would not hold over other sets of subjects. The only claim that they tested statistically was that recognition accuracy was impaired by familiarity. This claim is supported by all three experiments reported here. Ironically, Maddox and Estes (1997) found little influence of familiarity on accuracy in recognition. However, their analyses were restricted to items that had occurred on the familiarization series (i.e., those that had a familiarization frequency of at least one). The results of Experiments 1 and 2 here suggest that the largest change in accuracy results when familiarization frequency is increased from zero to one. Additional increases in familiarization frequency had only smaller effects on accuracy.

The empirical finding here was that both hits and false alarms increased as a function of familiarization frequency, with the latter showing a steeper increase. This finding is in agreement with results reported in recent studies. Dobbins, Kroll, Yonelinas, and Liu (1998, Experiment 1) have demonstrated that recognition accuracy declined significantly as a function of familiarization frequency; both hits and false alarms increased with higher levels of familiarization frequency, although no significance tests were reported on those data. Chalmers and Humphreys (1998) studied the effects of familiarization frequency on several different memory tests. In the conditions most similar to those studied here (a yes/no recognition test on either high-frequency words or very rare words, with the familiarization series and the study list presented on the same day), both hits and false alarms increased as a function of familiarization frequency, with the latter showing the greater increase.

It is likely that the pattern reported here must be subject to boundary conditions. For example, Chalmers and Humphreys (1998) found that, when a very low-frequency word was presented with its definition on each occurrence, accuracy in recognition increased as a function of familiarization frequency. For these stimuli (whose meanings were presumed to have been unknown to the subjects before the beginning of the experiment), additional opportunities to acquire the definitions were evidently beneficial, outweighing the negative effects that famil- iarization typically has on accuracy. Also, familiarization frequency had a much smaller effect on recognition if the familiarization series and the study list had been presented on separate days, presumably because subjects found it easier to discriminate between familiarization presentations and study-list presentations. Boundary conditions such as these make it clear that attempts to attribute recognition entirely to familiarity (defined operationally as frequency of occurrence) are doomed to failure.

Traditionally, studies of recognition memory have often been concerned with accuracy. However, a complete account of the processes underlying recognition will require an understanding of which manipulations lead to mirror effects (opposite effects on hits and false alarms) and which lead to concordant patterns (with hits and false alarms being affected in the same direction). The results reported here suggest that familiarity leads to a concordant pattern. This finding is consistent with the claim that familiarity plays an important role in recognition judgments.

\section{REFERENCES}

Chalmers, K. A., \& Humphreys, M. S. (1998). Role of generalized and episode specific memories in the word frequency effect in recognition. Journal of Experimental Psychology: Learning, Memory, \& Cognition, 24, 610-632.

Dobbins, I. G., Kroll, N. E. A., Yonelinas, A. P., \& LiU, Q. (1998). Distinctiveness in recognition and free recall: The role of recollection in the rejection of the familiar. Journal of Memory \& Language, 38 , $381-400$

EsTES, W. K. (1994). Classification and cognition. Oxford: Oxford University Press.

GiLlund, G., \& ShIFFrin, R. M. (1984). A retrieval model for both recognition and recall. Psychological Review, 91, 1-67.

GLANZER, M., \& ADAMS, J. K. (1985). The mirror effect in recognition memory. Memory \& Cognition, 13, 8-20.

HINTZMAN, D. L. (1988). Judgments of frequency and recognition memory in a multiple-trace model. Psychological Review, 95, 528-551.

MADDOX, W. T., \& EsTES, W. K. (1997). Direct and indirect stimulusfrequency effects in recognition. Journal of Experimental Psychology: Learning, Memory, \& Cognition, 23, 539-559.

ThorNDIKE, E. L., \& LoRGE, I. (1944). The teacher's wordbook of 30,000 words. New York: Columbia University, Teachers College, Bureau of Publications.

Tulving, E., \& Kroll, N. (1995). Novelty assessment in the brain and long-term memory encoding. Psychonomic Bulletin \& Review, 2, $387-390$.

(Manuscript received December 18, 1997; revision accepted for publication May 6, 1998 . 